\title{
Driving Liquid Chemistry with in situ STEM in Monolayer Window Encapsulated Liquid Cells
}

\author{
Jacob R. Jokisaari ${ }^{1}$, Arijita Mukherjee ${ }^{1}$, Xuan $\mathrm{Hu}^{1}$, and Robert Klie ${ }^{1}$ \\ ${ }^{1}$ University of Illinois at Chicago, Department of Physics, Chicago IL, 60607
}

There has long been considerable interest in imaging a liquid environment in TEM. Such capability has numerous applications as many different important processes involve liquid media, including corrosion, energy storage, and even organic and bio-systems [1]. Despite many developments allowing the use of liquid cells or hydrated samples in the vacuum environment required for electron microscopy, there remain some fundamental difficulties. One of these is the loss of resolution involved with imaging in liquid, for which there are several reasons. First, encapsulation of the liquid in the vacuum atmosphere necessitates the use of electron-transparent 'windows' which support the fluid yet allow the beam to penetrate. No truly transparent material exists; all will scatter the beam to some degree. Such windows are typically made of amorphous silicon nitride, and ideally, the thinner the window the better the transparency. However, limitations with modern lithographic processing methods and the thickness required to contain the internal pressure of the liquid cell require a thickness of approximately 30-100 $\mathrm{nm}$. The second factor reducing resolution is scattering of the electron beam by the fluid itself. This necessitates very thin liquid areas, but again, limitations of lithography make for larger-than-ideal liquid cell thickness.

Recently, considerable effort has been invested in using 2D materials for liquid cell windows, with some success using graphene, boron nitride, or other 2D monolayer materials [2]. In this case, the window is composed of pockets of water between a few sheets of graphene, creating a thin blister of liquid environment. In this case, the high strength and very thin $(<2 \mathrm{~nm}) 2 \mathrm{D}$ material encapsulates and stabilizes a very thin water droplet containing a test solution. Such droplets allow unprecedented atomic resolution imaging and spectroscopy of particles in liquid droplets and have been used to precipitate Au and Pt nanoparticles in-situ [2] and to encapsulate beam-sensitive biomaterials [3,4]. A schematic example of such a system is shown in Fig. 1.

An additional recent development in in situ liquid technique comes from greater understanding of the interaction between the electron beam and, for example, water [5]. It has been shown that the beam introduces active species in aqueous solutions, with electrolysis producing hydrogen and oxygen gases, solvated electrons, and similar. One application using the beam to control chemistry would be to use the dose rate to alter the $\mathrm{pH}$ of a solution [6]. Such control could be used to drive aqueous reactions, including dissolution/precipitation in $\mathrm{pH}$-sensitive materials. Combining the ability to control the $\mathrm{pH}$ with thin 2D material windows, dose-controlled reversible in-situ reactions can be examined utilizing the full imaging resolution of TEM as well as high quality EDS and EELS spectroscopy.

Samples consisting of 2D materials such as graphene were prepared and the electron beam was used to drive chemical changes. In the first case, we will discuss control of the $\mathrm{pH}$ via beam dose, quantified using high-resolution EELS of water and heavy water. The isotopic difference appears as a shift in the position of the EELS edge, and allows us to demonstrate the dose difference required for hydrolysis in these two cases. The shift in the edge position allows the $\mathrm{pH}$ change associated with electron beam irradiation to be somewhat quantified. We further apply this knowledge to drive $\mathrm{pH}$ dependent reactions in graphene liquid cells with an appropriate material such as hydroxyapatite (HAP), a common 
biomaterial. An example liquid cell of water with HAP particles showing beam-induced bubbles is shown in Fig. 2. In order to control the reactions, appropriate beam energy and alignment must be chosen to reduce beam damage to the specimen through careful choice accelerating voltage and a lowdose scanning pattern. These results demonstrate the world of new capabilities allowed by 2D window thin liquid cells and quantification of electron-beam/liquid interactions, which can be applied to questions in many different scientific fields.

\section{References:}

[1] Niels de Jonge and Frances M. Ross, Nature Nanotechnology, Vol 6, (2011) 695-704.

[2] Hellebusch, Michael F. Crommie, Jeong Yong Lee, A. Zettl, A. Paul Alivisatos, Jong Min Yuk, Jungwon Park, Peter Ercius, Kwanpyo Kim, Daniel J., Science, Vol 336, (2012) 61-64.

[3] Canhui Wang, Qiao Qiao, Tolou Shokuhfar, and Robert F. Klie, Adv. Mater., 26, (2014) 3410-3414. [4] Alexander J. Donovan, Joseph Kalkowski, Magdalena Szymusiak, Canhui Wang, Stephanie A.

Smith, Robert F. Klie, James H. Morrissey, and Ying Liu, Biomacromolecules, 17, (2016) 2572-2581. [5] Joseph M. Grogan, Nicholas M. Schneider, Frances M. Ross, and Haim H. Bau, Nano Lett., Vol 14, (2014) 359-364.

[6] Nicholas M. Schneider, Michael M. Norton, Brian J. Mendel, Joseph M. Grogan, Frances M. Ross, and Haim H. Bau, J. Phys. Chem. C, 118, (2014) 22373-22382.

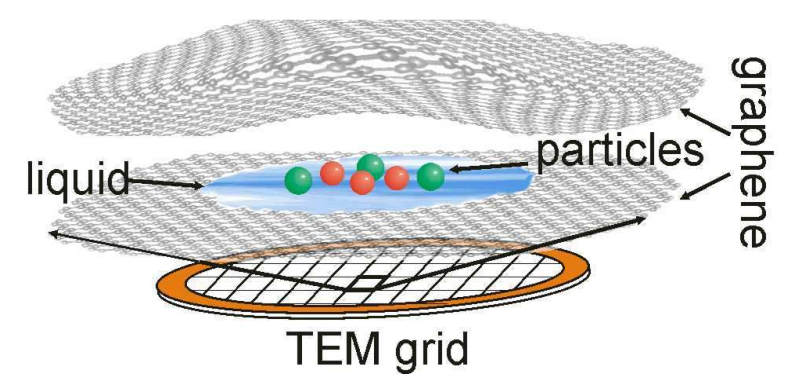

Figure 1. Schematic of 2D liquid cell

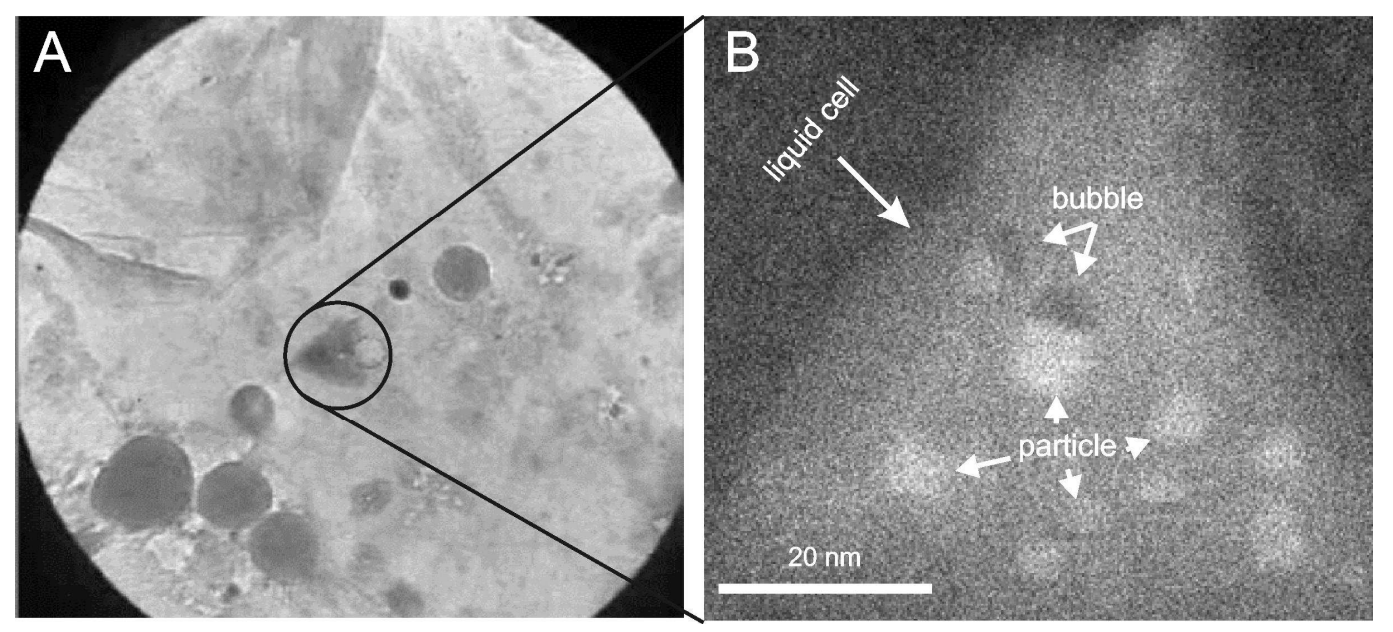

Figure 2. (A) Ronchigram image of graphene liquid cell showing several LCs (B) HAADF image of liquid cell showing beam-induced bubbles and hydroxyapatite particles 